\title{
Absence of proton tunneling during the hydrogen-bond symmetrization in $\delta$-AlOOH
}

\author{
Florian Trybel $\odot,{ }^{1,2, *}$ Thomas Meier $\odot,{ }^{1,3}$ Biao Wang $\odot,{ }^{1,4}$ and Gerd Steinle-Neumann $\odot^{1}$ \\ ${ }^{1}$ Bayerisches Geoinstitut, Universität Bayreuth, D-95440 Bayreuth, Germany \\ ${ }^{2}$ Department of Physics, Chemistry and Biology (IFM), Linköping University, SE-581 83 Linköping, Sweden \\ ${ }^{3}$ Center for High Pressure Science and Technology Advanced Research (HPSTAR), Beijing 100094, China \\ ${ }^{4}$ Department of Earth Sciences, University of Oxford, OX1 3AN Oxford, United Kingdom
}

(Received 28 June 2021; revised 11 August 2021; accepted 26 August 2021; published 24 September 2021)

\begin{abstract}
$\delta$-AlOOH is of significant crystallochemical interest due to a subtle structural transition near $10 \mathrm{GPa}$ from a $P 2{ }_{1} \mathrm{~nm}$ to a Pnnm structure, the nature and origin of hydrogen disorder, the symmetrization of the $\mathrm{O}-\mathrm{H} \cdots \mathrm{O}$ hydrogen bond and their interplay. We perform a series of density functional theory-based simulations in combination with high-pressure nuclear magnetic resonance (NMR) experiments on $\delta$-AlOOH up to $40 \mathrm{GPa}$ with the goal to better characterize the hydrogen potential and therefore the nature of hydrogen disorder. Simulations predict a phase transition in agreement with our NMR experiments at $10-11 \mathrm{GPa}$ and hydrogen bond symmetrization at $14.7 \mathrm{GPa}$. Calculated hydrogen potentials do not show any double-well character and there is no evidence for proton tunneling in our NMR data.
\end{abstract}

DOI: 10.1103/PhysRevB.104.104311

\section{INTRODUCTION}

Hydrogen is an important chemical component in the Earth's mantle, as even a small amount can strongly affect key properties of minerals, such as melting temperature, rheology, electrical conductivity, and atomic diffusion [1-4]. Therefore, over the past 20 years, many hydrous minerals, such as dense hydrous magnesium silicates [5], have been synthesized at high-pressure $(P)$ and high-temperature $(T)$ conditions and investigated as potential candidates for hydrogen transport to the lower mantle. However, most of these minerals decompose at $P<60 \mathrm{GPa}$, where phase $\mathrm{H}$ breaks down to $\mathrm{MgSiO}_{3}$ bridgmanite and a fluid component [6-8].

In 2017, $\mathrm{AlSiO}_{3}(\mathrm{OH})$ was found in diamond inclusions [9] from the mantle transition zone at a depth of $410-660 \mathrm{~km}$. High- $P$, high- $T$ experiments revealed that this phase can form from hydrous sediment components at upper mantle conditions (10-12 GPa) [10] and decomposes to $\delta$-AlOOH and $\mathrm{SiO}_{2}$ stishovite at conditions similar to those found at the base of the mantle transition zone $(P>20 \mathrm{GPa})[11] . \delta$-AlOOH is particularly interesting as it shows a wide stability range including conditions along the geotherm of a subducting slab [12-14] and may therefore be a potential host of hydrogen in Earth's lower mantle.

$\delta$-AlOOH crystallizes in a primitive orthorhombic lattice with space group $P 2{ }_{1} n m$ at ambient conditions [12,15-17]. Its

\footnotetext{
*florian.trybel@liu.se
}

Published by the American Physical Society under the terms of the Creative Commons Attribution 4.0 International license. Further distribution of this work must maintain attribution to the author(s) and the published article's title, journal citation, and DOI. Funded by Bibsam. structure corresponds to distorted rutile, with $\mathrm{Al}$ and $\mathrm{O}$ atoms located on mirror planes (Fig. 1). $\mathrm{AlO}_{6}$ octahedra share edges along the $c$ axis and these octahedra chains are connected via corners (Fig. 1). There are two distinct oxygen positions (O1 and $\mathrm{O} 2)$ at the vertices and in the equatorial plane, respectively. Layers of $\mathrm{AlO}_{6}$ octahedra oriented in different directions are connected with an asymmetric hydrogen bond between the layers.

Single-crystal synchrotron x-ray diffraction (XRD) $[15,18]$ and neutron diffraction measurements $[19,20]$ identified a sub- to supergroup phase transition from $P 2{ }_{1} n m$ to Pnnm at $\sim 10 \mathrm{GPa}$ where $\mathrm{O} 1$ and $\mathrm{O} 2$ positions become equivalent. The transition is accompanied by a rotation of the $\mathrm{AlO}_{6}$ octahedra by $\sim 1^{\circ}$, and in the same $P$ range the hydrogen position is predicted to symmetrize [18,20-23]. In Fourier difference maps calculated from their neutron diffraction data, Sano-Furukawa et al. [20] found a bimodal hydrogen distribution between 9.5 and $18 \mathrm{GPa}$, supporting previous suggestions $[18,21,22]$ that a double-well potential along the diagonal O-O direction may exist, which could give rise to proton tunneling, similar to the state found during the ice-VII to ice-X transition [24-26]. No direct evidence of tunneling has been found to date, however.

We investigate the phase transition, hydrogen bond symmetrization (a central unimodal proton distribution between the two respective oxygen atoms), and the possibility of proton tunneling in $\delta-\mathrm{AlOOH}$, combining density functional theory (DFT)-based calculations and high- and low-field high$P$ nuclear magnetic resonance (NMR) spectroscopy. With DFT, we perform a stepwise optimization of the host lattice and the hydrogen positions over a wide volume $(V)$ range and analyze the potential seen by the hydrogen atoms as well as the geometry of the $\mathrm{AlO}_{6}$ octahedra. We analyze the signal shift as well as the full width at half maximum (FWHM) of our high-field NMR experiments at $P$ of up to $40 \mathrm{GPa}$ (c.f. Supplemental Fig. S1 in the Supplemental Material [27]), 
searching for characteristic features of a phase transition and using low-field NMR data at $5.6 \mathrm{GPa}$, we investigate indications of proton tunneling [25].

\section{COMPUTATIONAL DETAILS}

All DFT simulations are performed using the Quantum ESPRESSO package [28,29], where possible GPU-accelerated [30]. We use projector augmented wave atomic files for $\mathrm{Al}, \mathrm{O}$, and $\mathrm{H}$ based on the PBEsol [31] approximation to exchangecorrelation which was previously found to show excellent agreement with experimental data [22]. For $\mathrm{Al}$ the $2 \mathrm{p}$ electrons and lower and for $\mathrm{O}$ the $1 \mathrm{~s}$ electrons are treated as semirelativistic core states. Convergence tests with a threshold of $10^{-5} \mathrm{Ry} /$ atom lead to a Monkhorst-Pack k-point grid [32] of $12 \times 8 \times 8$ for primitive unit cells and a cutoff energy for the plane-wave expansion of 140 Ry. We use the FINDSYM code [33] for symmetry analysis and VESTA 3 [34] for visualization of structures.

\section{EXPERIMENTAL DETAILS}

High- $P$ cells with pairs of $250-\mu \mathrm{m}$ culet diamond anvils are used, and the preparation of the NMR experiments closely follows the procedure outlined in our previous work [35]; $3.2 \mathrm{~mm}$ excitation coils are formed from single turn cover inductors made from $50 \mu \mathrm{m}$ of copper-coated teflon foil. The diamond anvil is coated with $1 \mu \mathrm{m}$ of copper using physical vapor deposition, and subsequently Lenz lens resonators are cut out of this layer using focused ion beam milling.

A $20-\mu \mathrm{m}^{3}$ crystal of $\delta$ - $\mathrm{AlOOH}$ (synthesis is described in Ref. [36]) is placed in the sample chamber and the DAC is filled with neon as a $P$-transmitting medium. Both excitation coils are mounted central to the diamond anvils and connected in a Helmholtz coil arrangement after closing the cell. For the high-field measurements, $1045 \mathrm{mT}$ with a corresponding ${ }^{1} \mathrm{H}$ resonance frequency of $\sim 45 \mathrm{MHz}$ in an electromagnet is used. Additional homonuclear ${ }^{1} \mathrm{H}-{ }^{1} \mathrm{H}$ decoupling experiments are conducted using a Lee-Goldburg [37] saturation pulse of $25 \mathrm{~W}$ prior to the spin excitation to obtain high-resolution ${ }^{1} \mathrm{H}-\mathrm{NMR}$ spectra. An additional DAC prepared in a similar manner is filled with distilled $\mathrm{H}_{2} \mathrm{O}$ and used as a resonance shift reference. Low-field measurements are performed at $125 \mathrm{mT}$ and a resonance frequency of $\sim 5 \mathrm{MHz}$, using the same electromagnet. Resulting spectra are analyzed by line form matching to the experimental signal [38]. Pressure is calculated from the Raman signal of the diamonds $[39,40]$ and the $\mathrm{AlOOH}$ volume using the equation-of-state (EOS) from Simonova et al. [36].

\section{STRUCTURAL OPTIMIZATION AND HYDROGEN POTENTIAL}

In the computations, we start structural optimization with reported experimental low- $P$ structures from Komatsu et al. [15] and Sano-Furukawa et al. [20] and optimize the hydrogen position for $V$ between 57 and $47 \AA^{3}$. We sample and optimize the hydrogen positions along the diagonal oxygen-oxygen direction first, relax the coordinates of all atoms, perform a second sampling and optimization of the hydrogen positions,

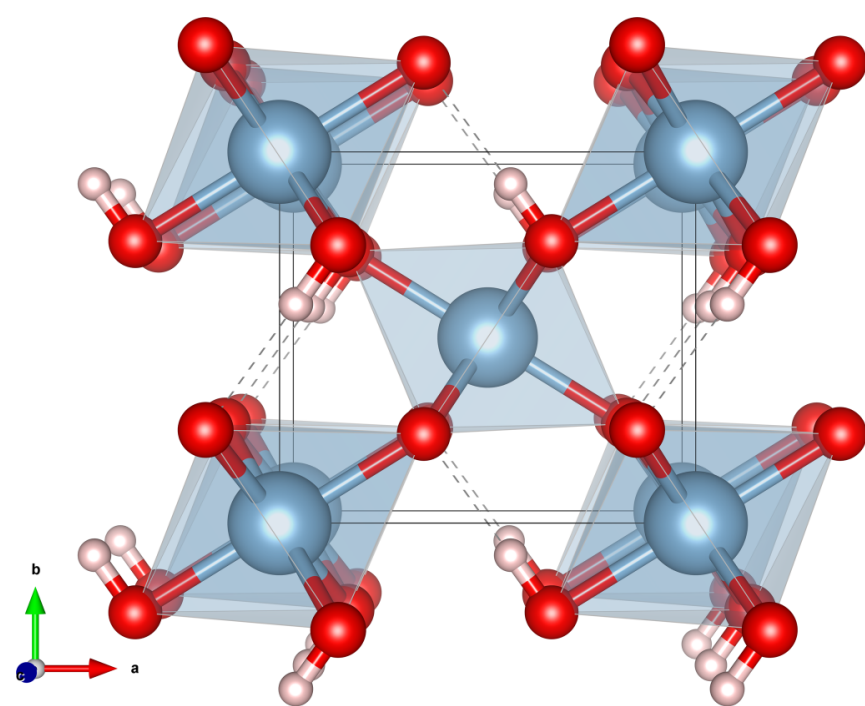

FIG. 1. Crystal structure of $P 2{ }_{1} n m \delta$-AlOOH. Aluminum is shown in blue, oxygen in red, and hydrogen in white. Offset of the hydrogen positions from the center is exaggerated for illustration.

relax the cell parameters, and perform a final sampling and optimization of the hydrogen positions. Both initial structures $[15,20]$ converge to the same coordinates within the first steps of the relaxation.

We sample configurations by displacing the hydrogen atoms along the diagonal oxygen-oxygen direction in the $a-b$ plane by starting with both hydrogen atoms being close to the respective oxygen atom with the smaller $b$ coordinate (normal configuration) and from the configuration shown in Fig. 1 (inverse configuration, c.f. Supplemental Fig. S2 of the Supplemental Material [27]). From the energy obtained in each sampling step, we construct the potential seen by the hydrogen atom, similar to our previous work on the ice-VII to ice-X transition [26] via spline interpolation at each sampled $V$ for both configurations. The final positions of the hydrogen atoms are obtained as the minima of the respective spline interpolation (Fig. 2).

We find distinctly different potential symmetries for normal and inverse sampling with respect to the center of the diagonal oxygen-oxygen distance: Normal sampling results in a symmetric potential; inverse sampling results in an asymmetric potential ( $\sim 0.2 \mathrm{eV}$ lower in energy). Under compression the potentials become narrower in both sampling types, and for inverse sampling asymmetry decreases. At $V \lesssim 52.4 \pm 0.1 \AA^{3}$ the potential obtained by inverse sampling becomes symmetric and the energy difference between the two potentials approaches zero, with the inverse sampling remaining slightly lower in energy over the full $V$ range.

Contradicting prior suggestions [21,22], both potentials do not show any double-well character, even though calculating the potential seen by the hydrogen atom from Kohn-Sham DFT should strongly overestimate the potential well without further consideration of the quantum nature of the hydrogen atoms $[22,26,41]$.

After each optimization step, we analyze the space group of the resulting cell and track the rotation of $\mathrm{AlO}_{6}$ octahedra 


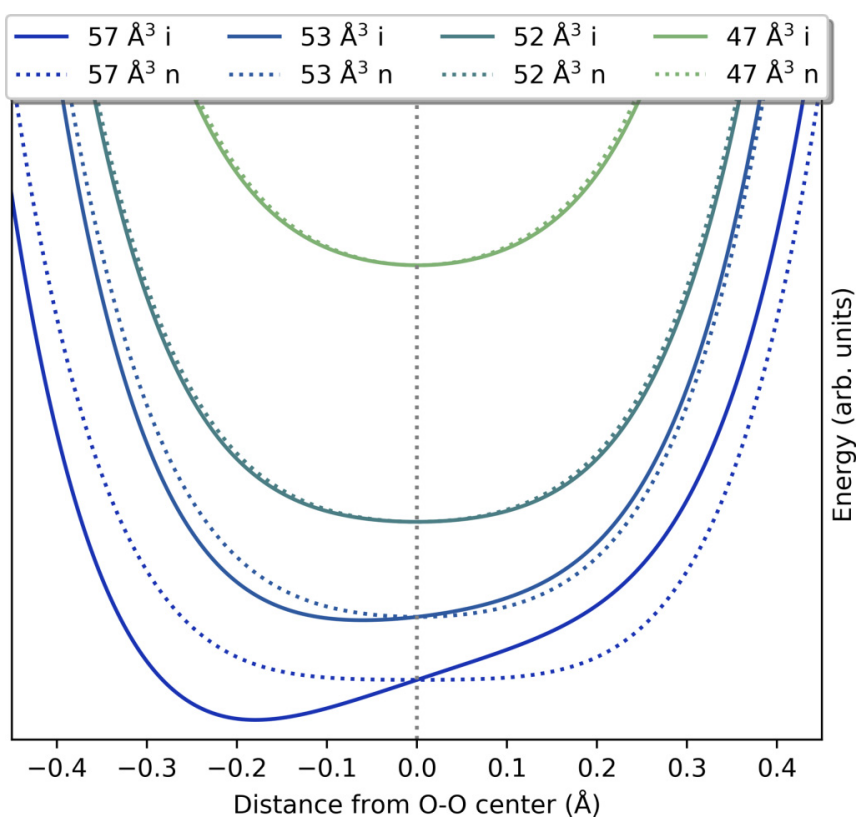

FIG. 2. Comparison of normal (dotted) and inverse (solid) sampling (Supplemental Fig. S2 in the Supplemental Material [27]) of the potential seen by the hydrogen atom. At large volumes (low $P$ ), normal sampling leads to a symmetric and inverse sampling to an asymmetric single-well potential with a significantly lower energy $(\sim 0.2 \mathrm{eV})$. With increasing compression, this difference vanishes as the two oxygen positions become equivalent. By construction, the potentials are equivalent at the central position (indicated by the vertical dashed line).

using angles $\omega$ and $\omega^{\prime}$ as defined by Sano-Furukawa et al. [20] as a function of compression (Fig. 3).

We find an increase in the angle of $\sim 0.6^{\circ}$ when compressing from $57 \AA^{3}$ to $\sim 53 \AA^{3}$, followed by a decrease at higher compression for both $\omega$ and $\omega^{\prime}$. The angles are in general $\sim 0.5^{\circ}$ larger than the experimental values by Sano-Furukawa et al. [20] which show large scatter; the difference between $\omega$ and $\omega^{\prime}$ and the $P$ dependence they report are in very good agreement with our calculations.

\section{NMR SPECTROSCOPY}

NMR experiments employing Lee-Goldburg decoupling pulses lead to line widths of $\sim 1.5 \mathrm{ppm}$ [shown for 8 and $11 \mathrm{GPa}$ in Fig. 4(a)] which permits the analysis of chemical shifts with $\sim 10 \mathrm{ppm}[$ (Fig. 4(c)]. For the chemical shift, we find an abrupt change at $P=9.9 \pm 0.6 \mathrm{GPa}$ from $\sim 5$ to $>12 \mathrm{ppm}$, indicating a structural change in the chemical environment of the hydrogen atoms as expected for a phase transition. We find a single proton signal over the full pressure range indicating a unique, geometrically well-defined proton position, in agreement with an earlier NMR study at ambient conditions by Xue and Kanzaki [42].

Lee-Goldburg decoupling, while retaining information about the isotropic chemical shift interactions and strongly focusing the line width, leads to a cancellation of the nonsecular parts of the total spin Hamiltonian and thus to a loss of information about spin interactions due to chemical shift anisotropy, direct homo- and heteronuclear dipole-dipole, as

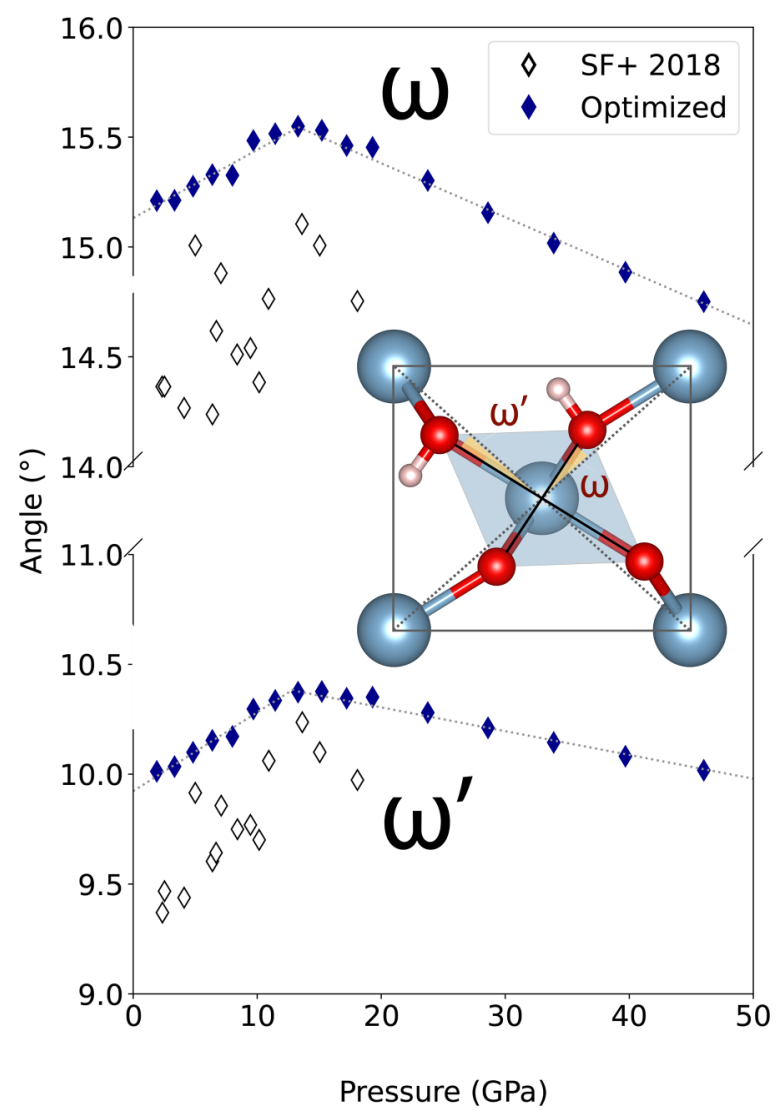

FIG. 3. Angle analysis for the fully relaxed cell (filled symbols) and the experimental values by Sano-Furukawa et al. [20] (open symbols, SF+2018) following their definition (inset).

well as first-order quadrupolar interactions. Therefore, we additionally analyze the line shape of ${ }^{1} \mathrm{H}-\mathrm{NMR}$ solid-echoes (Supplemental Fig. S1 in the Supplemental Material [27]) and find a minimum in the line width at $P=12.7 \pm 0.4 \mathrm{GPa}$ (Fig. 4(d)), indicating a change in proton mobility.

Proton tunneling should lead to a zero-field splitting accompanied by detectable tunneling side bands as it introduces an exchange between allowed magnetic transitions with $\Delta m=1$ and usually forbidden transitions [43]. In work on the ice-VII to ice-X transition in high- $P \mathrm{H}_{2} \mathrm{O}$ [25], we showed that such tunneling side bands can be resolved at high $P$ using low-field NMR, and that NMR is sensitive to changes in the tunneling rate during compression. We therefore employ the low-field setup at $P=5.6 \mathrm{GPa}$ (Fig. 4(b)) and find no indication for tunneling sidebands in $\delta-\mathrm{AlOOH}$, in agreement with the $P$ evolution of the calculated potentials, showing no double-well character over the respective compression range.

\section{EQUATION-OF-STATE}

In order to convert $V$ of the simulation cells to $P$, we use the optimized structures from the inverse configuration and fit a third-order Birch-Murnaghan (BM3) EOS to total energy. We use the $V$ at which the potential symmetrizes $\left(52.4 \AA^{3}\right)$ to split the $E-V$ results in two sets and calculate an EOS for (i) the full $V$ range, (ii) $57 \geqslant V \geqslant 53.5 \AA^{3}$, and (iii) $52 \geqslant$ $V \geqslant 47 \AA^{3}$ (Fig. 5(a)). We calculate the intersection of the 

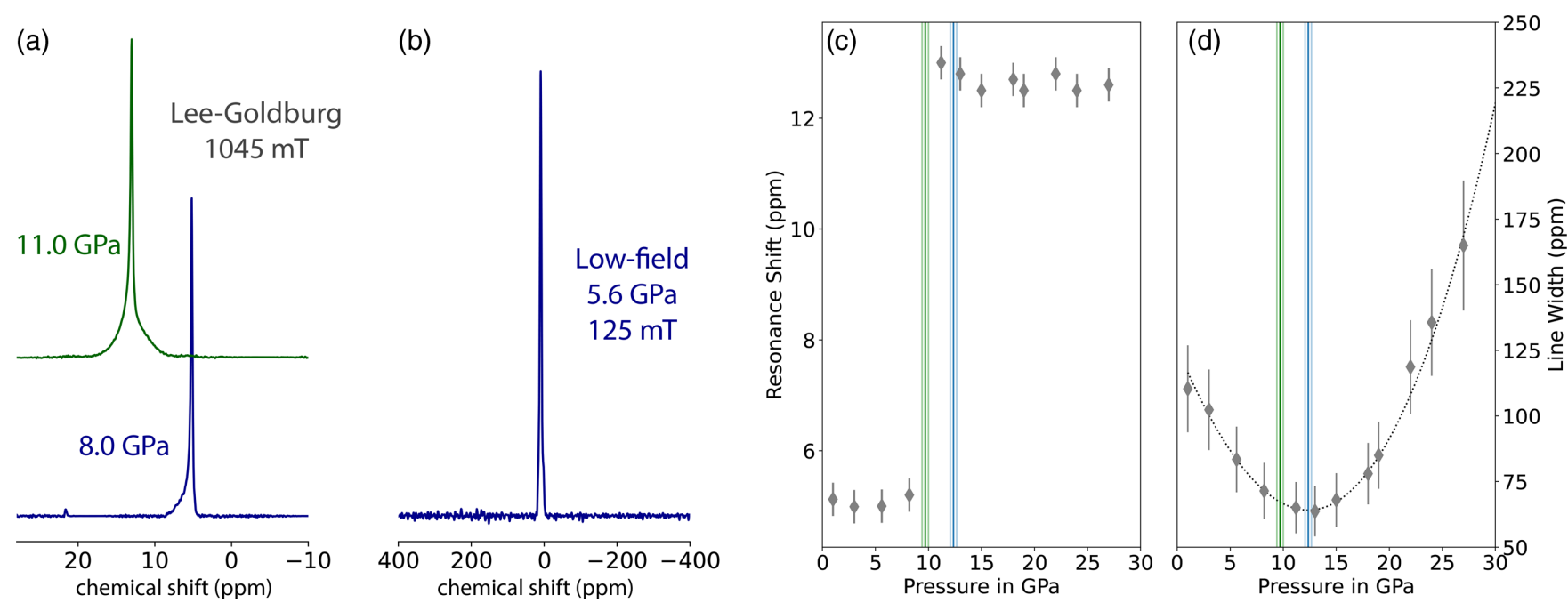

FIG. 4. (a) Lee-Goldburg ${ }^{1} \mathrm{H}-\mathrm{NMR}$ spectra at 8 and $11 \mathrm{GPa}$ at a field of $1045 \mathrm{mT}$ with $\mathrm{H}_{2} \mathrm{O}$ as a chemical shift reference. (b) NMR spectrum of $\delta$-AlOOH at $5.6 \mathrm{GPa}$ (shifted to zero) at a field of $125 \mathrm{mT}$. (c) Shift of the resonance as a function of pressure in the Lee-Goldburg spectra. The shift increases abruptly at $P=9.9 \pm 0.6 \mathrm{GPa}$ from $\sim 5 \mathrm{ppm}$ to $>12 \mathrm{ppm}$. (d) FWHM of the resonance peak as a function of $P$. A minimum in line width occurs at $P=12.7 \pm 0.4 \mathrm{GPa}\left(\sim 52.7 \AA^{3}\right)$. The dotted black line is a third-order polynomial fit to the data between 5 and $20 \mathrm{GPa}$. In panels (c) and (d), the blue vertical lines show the minima of the polynomial fit to the line width data and the green line indicates the jump in the resonance shift with the respective errors indicated by the dotted lines.

enthalpy curves for (ii) and (iii) and estimate the transition $P$ as $11.3 \pm 0.6 \mathrm{GPa}$ [horizontal line in Fig. 5(a)], where the error is estimated from the shift when the point closest to the transition in the potential is in-/excluded from the respective $V$ ranges. The phase transition is of second order as we do not find a $V$ collapse.
The calculated EOS parameters (low $P: V_{0}=56.0 \AA^{3}$, $K_{0}=183 \mathrm{GPa}, K_{0}^{\prime}=3.7$; high $P: V_{0}=55.5 \AA^{3}, K_{0}=$ $\left.224 \mathrm{GPa}, K_{0}^{\prime}=4.0\right)$ are in good agreement with partial EOS parameters by Simonova et al. [36] (low $P: V_{0}=56.51(8) \AA^{3}$, $K_{0}=142(5) \mathrm{GPa}$; high- $P$ part $V_{0}=55.56(8) \AA^{3}, K_{0}=$ 216.0(5) GPa, with $K_{0}^{\prime}=4.0$ in both cases) and data from

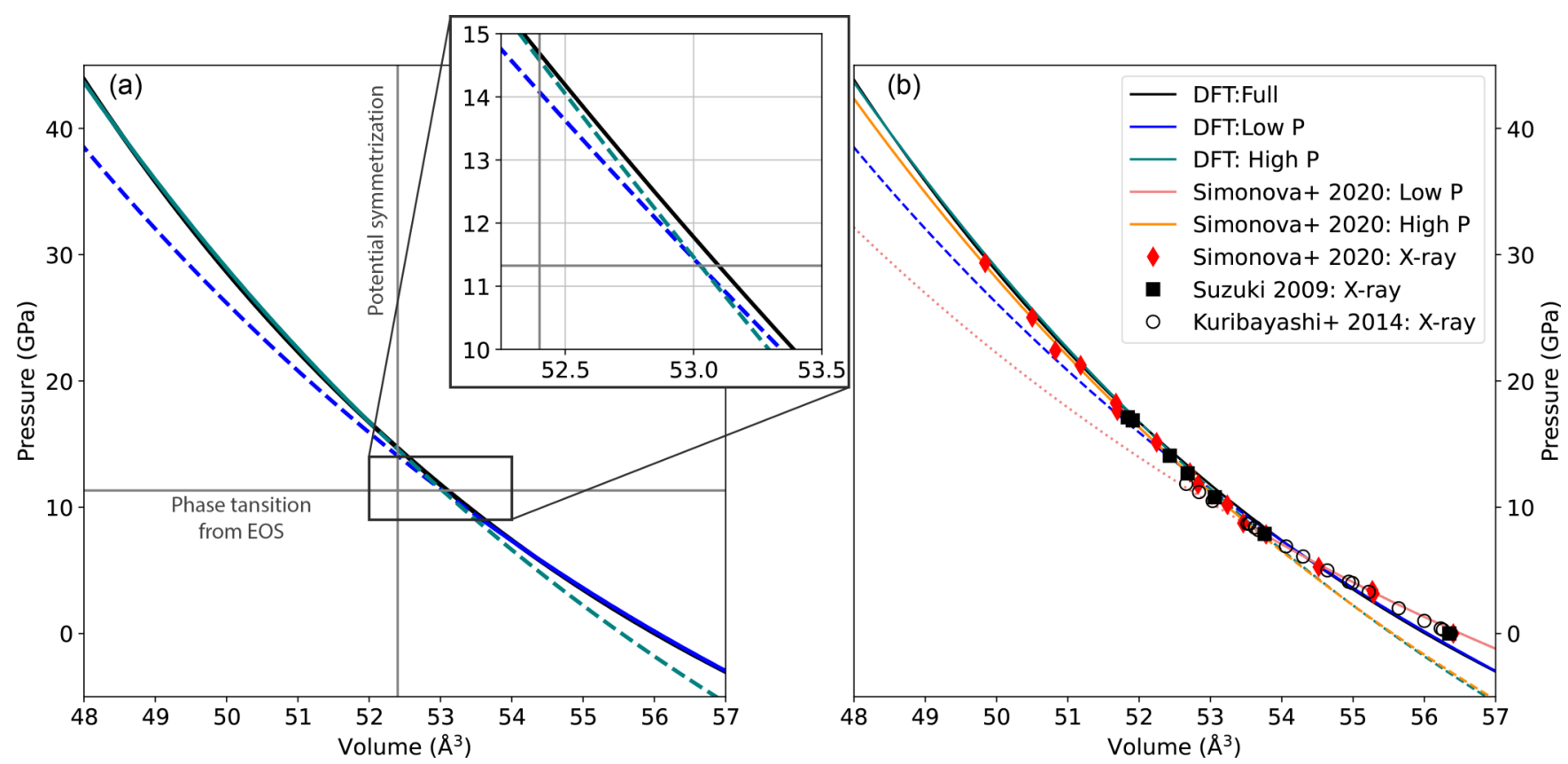

FIG. 5. (a) Third order Birch-Murnaghan (BM3) EOS fit to the total energy of the optimized structures from the inverse configuration sampling. The full $V$ range is fitted by the black curve, $V>53.5 \AA^{3}$ by the blue curve, and $V<52 \AA^{3}$ by the teal curve. The vertical line shows the estimated transition from the potential analysis, and the horizontal line is the intersection of the enthalpy calculated from the two partial BM3 fits. The inset shows a zoom of the area where low- and high- $P$ partial EOS intersect. (b) Comparison of different $V-P$ data and EOS from our calculations with literature data by Kuribayashi et al. [18], Simonova et al. [36], and Suzuki [44]. 
TABLE I. Calculated values of the transition pressure from the low- $P P 2_{1} n m$ to the high- $P$ Pnnm structure of AlOOH. For the line width analysis, errors are estimated from the $P$ error and for the chemical shift from the $P$ resolution of the measurement. For the simulations, errors are estimated from the $P$ resolution of the sampling and for the EOS calculation as the difference between the intersection $P$ of the low- $P$ and the high- $P$ sub-EOS (Fig. 5). The last three columns show literature data from Simonova et al. [36] (S+ 2020), Sano-Furukawa et al. [20] $(\mathrm{SF}+2018)$, and Pillai et al. $[21](\mathrm{P}+2018)$.

\begin{tabular}{ccccccccc}
\hline \hline Criterion & NMR Shift & EOS & $<$ AlOAl $>$ angles & NMR line width & Potential & S +2020 & SF+ 2018 & P +2018 \\
\hline$P(\mathrm{GPa})$ & $9.9 \pm 0.6$ & $11.3 \pm 0.6$ & $13.1 \pm 0.7$ & $12.4 \pm 0.3$ & $14.7 \pm 0.4$ & $>10$ (structural) & $9 / 18.1$ & $8 / 15$ \\
\hline \hline
\end{tabular}

Suzuki [44] and Kuribayashi et al. [18] at $V<55 \AA^{3}$ (Fig. 5(b)). At larger $V$, our EOS slightly underestimates $P$ compared with the experimental EOS, which is most likely caused by thermal effects not being included in the calculations.

Using the respective low- $P$ and high- $P$ EOS parameters, we convert all $V$ dependencies to $P$ dependencies and find potential symmetrization at $P=14.7 \pm 0.4 \mathrm{GPa}$ and the maxima in $\omega$ and $\omega^{\prime}$ at $P=13.1 \pm 0.7 \mathrm{GPa}$ (Table I).

\section{DISCUSSION AND CONCLUSION}

We have analyzed different properties of $\delta$-AlOOH that can be directly linked to the phase transition (chemical shift, EOS) and hydrogen bond symmetrization (potential symmetry), but also properties that should record an influence of both processes (angles $\omega$ and $\omega^{\prime}$ as well as the NMR line width). We find features in the same three $P$ regions: (i) The phase transition characterized by the change in chemical shift at $9.9 \pm 0.6 \mathrm{GPa}$ and the change in the slope of the $E-V$ curve (and therefore splitting of the EOS) at $11.3 \pm 0.4 \mathrm{GPa}$; (ii) a maximum in the angles $\omega$ and $\omega^{\prime}$ at $13.1 \pm 0.7 \mathrm{GPa}$ and a minimum in the line width at $12.4 \pm 0.3 \mathrm{GPa}$; (iii) the symmetrization of the potential at $14.7 \pm 0.4 \mathrm{GPa}$.

Comparing our results with recently published experiments $[20,36]$ and computations $[21,22]$, we find that we match the phase transition measured via neutron diffraction by SanoFurukawa et al. [20] and XRD by Simonova et al. [36] at $\sim 10 \mathrm{GPa}$ in the NMR experiments and the calculations. Furthermore, we reproduce the hydrogen bond symmetrization estimate from the calculation of elastic constants by Cortona [22] and Pillai et al. [21] at $\sim 15 \mathrm{GPa}$ in our potential analysis. For angles $\omega$ and $\omega^{\prime}$, we find the same increase in slope as Sano-Furukawa et al. [20], with a maximum at $13.1 \pm$ $0.7 \mathrm{GPa}$. If we take a closer look at the data by Sano-Furukawa et al. [20], values for the angles $\omega$ and $\omega^{\prime}$ at the highest three
$P$ points $(P \geqslant 13 \mathrm{GPa})$ appear to decrease linearly with $P$, in agreement with our computational results (Fig. 3). The large scatter and limited $P$ range in the experimental data impede a more detailed analysis, comparison, and discussion, however.

The absence of a double well in our mapping of the hydrogen potential at any $V$ is supported by low-field NMR measurements which do not show any indication of tunneling side bands. Therefore, we conclude that, contrary to the iceVII to ice-X transition [25,26], there is no tunneling-induced proton disorder in $\delta$ - $\mathrm{AlOOH}$. The only observation directly linked to a double-well potential are the Fourier difference maps by Sano-Furukawa et al. [20] that describe an asymmetric proton distribution at $P>9.5 \mathrm{GPa}$, followed by a bimodal distribution to $P \lesssim 15 \mathrm{GPa}$ and a symmetric unimodal distribution at $P=18 \widetilde{\mathrm{GPa}}$. According to our results and data, the intermediate (bimodal) state is not characterized by proton disorder. Rather, it reflects order with weak asymmetry that gradually decreases. Therefore, we suggest the following interpretation of the neutron data: $\mathrm{As} \mathrm{O} 1$ and $\mathrm{O} 2$ become symmetrically equivalent during the structural transition from $P 2_{1} n m$ to Pnnm at $\sim 10 \mathrm{GPa}$, asymmetry can no longer be associated to an $\mathrm{O} 1-\mathrm{H} \cdots \mathrm{O} 2$ bond, but an averaged picture emerges where protons are closer to former $\mathrm{O} 1$ and $\mathrm{O} 2$ atoms, which may be visible in the Fourier difference maps and lead to the bimodal distribution if projected onto a Pnnm unit cell.

\section{ACKNOWLEDGMENTS}

F.T. and G.S.-N. were supported by Deutsche Forschungsgemeinschaft (DFG) within FOR 2440 (Matter under Planetary Interior Conditions) with Grant STE1105/13-1 and T.M. with Grant ME5206/3-1. F.T. was further supported by the Swedish Research Council (VR) Grant No. 2019-05600. The authors thank Niccolò Satta and Giacomo Criniti for very helpful discussions. Computations were partly performed at the Leibniz Supercomputing Centre of the Bavarian Academy of Sciences and the Humanities. GPU accelerated computations are supported via the NVIDIA Hardware Grant.
[1] D. Bercovici and S.-i. Karato, Nature (London) 425, 39 (2003).

[2] T. Inoue, Phys. Earth Planet. Inter. 85, 237 (1994).

[3] S.-I. Karato, M. S. Paterson, and J. D. FitzGerald, J. Geophys. Res.: Solid Earth 91, 8151 (1986).

[4] T. Yoshino, T. Matsuzaki, S. Yamashita, and T. Katsura, Nature (London) 443, 973 (2006).

[5] A. B. Thompson, Nature (London) 358, 295 (1992).

[6] E. Ohtani, Y. Amaike, S. Kamada, T. Sakamaki, and N. Hirao, Geophys. Res. Lett. 41, 8283 (2014).
[7] J. Tsuchiya and K. Umemoto, Geophys. Res. Lett. 46, 7333 (2019).

[8] M. Nishi, J. Tsuchiya, T. Arimoto, S. Kakizawa, T. Kunimoto, Y. Tange, Y. Higo, and T. Irifune, Phys. Chem. Miner. 45, 995 (2018).

[9] R. Wirth, C. Vollmer, F. Brenker, S. Matsyuk, and F. Kaminsky, Earth Planet. Sci. Lett. 259, 384 (2007).

[10] S. Ono, J. Geophys. Res.: Solid Earth 103, 18253 (1998).

[11] A. Sano, E. Ohtani, T. Kubo, and K.-i. Funakoshi, J. Phys. Chem. Solids 65, 1547 (2004). 
[12] A. Sano, E. Ohtani, T. Kondo, N. Hirao, T. Sakai, N. Sata, Y. Ohishi, and T. Kikegawa, Geophys. Res. Lett. 35, L03303 (2008).

[13] Y. Duan, N. Sun, S. Wang, X. Li, X. Guo, H. Ni, V. B. Prakapenka, and Z. Mao, Earth Planet. Sci. Lett. 494, 92 (2018).

[14] X. Su, C. Zhao, C. Lv, Y. Zhuang, N. Salke, L. Xu, H. Tang, H. Gou, X. Yu, Q. Sun, and J. Liu, Geosci. Front. 12, 937 (2021).

[15] K. Komatsu, T. Kuribayashi, A. Sano, E. Ohtani, and Y. Kudoh, Acta Crystallogr., Sect. E: Struct. Rep. Online 62, i216 (2006).

[16] E. Ohtani, K. Litasov, A. Suzuki, and T. Kondo, Geophys. Res. Lett. 28, 3991 (2001).

[17] A. Suzuki, E. Ohtani, and T. Kamada, Phys. Chem. Miner. 27, 689 (2000).

[18] T. Kuribayashi, A. Sano-Furukawa, and T. Nagase, Phys. Chem. Miner. 41, 303 (2014).

[19] A. Sano-Furukawa, K. Komatsu, C. B. Vanpeteghem, and E. Ohtani, Am. Mineral. 93, 1558 (2008).

[20] A. Sano-Furukawa, T. Hattori, K. Komatsu, H. Kagi, T. Nagai, J. J. Molaison, A. M. dos Santos, and C. A. Tulk, Sci. Rep. 8, 15520 (2018).

[21] S. B. Pillai, P. K. Jha, A. Padmalal, D. Maurya, and L. Chamyal, J. Appl. Phys. 123, 115901 (2018).

[22] P. Cortona, J. Phys.: Condens. Matter 29, 325505 (2017).

[23] J. Tsuchiya, T. Tsuchiya, S. Tsuneyuki, and T. Yamanaka, Geophys. Res. Lett. 29, 15 (2002).

[24] L. Lin, J. A. Morrone, and R. Car, J. Stat. Phys. 145, 365 (2011).

[25] T. Meier, S. Petitgirard, S. Khandarkhaeva, and L. Dubrovinsky, Nat. Commun. 9, 2766 (2018).

[26] F. Trybel, M. Cosacchi, T. Meier, V. M. Axt, and G. SteinleNeumann, Phys. Rev. B 102, 184310 (2020).

[27] See Supplemental Material at http://link.aps.org/supplemental/ 10.1103/PhysRevB.104.104311 for an additional visualization of the structures and ${ }^{1} \mathrm{H}-\mathrm{NMR}$ solid-echoes.

[28] P. Giannozzi, S. Baroni, N. Bonini, M. Calandra, R. Car, C. Cavazzoni, D. Ceresoli, G. L. Chiarotti, M. Cococcioni, I. Dabo, A. Dal Corso, S. de Gironcoli, S. Fabris, G. Fratesi, R. Gebauer, U. Gerstmann, C. Gougoussis, A. Kokalj, M. Lazzeri, L. Martin-Samos et al., J. Phys.: Condens. Matter 21, 395502 (2009).
[29] P. Giannozzi, O. Andreussi, T. Brumme, O. Bunau, M. B. Nardelli, M. Calandra, R. Car, C. Cavazzoni, D. Ceresoli, M. Cococcioni, N. Colonna, I. Carnimeo, A. D. Corso, S. de Gironcoli, P. Delugas, R. A. D. Jr., A. Ferretti, A. Floris, G. Fratesi, G. Fugallo et al., J. Phys.: Condens. Matter 29, 465901 (2017).

[30] J. Romero, E. Phillips, G. Ruetsch, M. Fatica, F. Spiga, and P. Giannozzi, in International Workshop on Performance Modeling, Benchmarking and Simulation of High Performance Computer Systems (Springer, Cham, 2017), pp. 67-87.

[31] J. P. Perdew, A. Ruzsinszky, G. I. Csonka, O. A. Vydrov, G. E. Scuseria, L. A. Constantin, X. Zhou, and K. Burke, Phys. Rev. Lett. 100, 136406 (2008).

[32] H. J. Monkhorst and J. D. Pack, Phys. Rev. B 13, 5188 (1976).

[33] H. T. Stokes and D. M. Hatch, J. Appl. Crystallogr. 38, 237 (2005).

[34] K. Momma and F. Izumi, J. Appl. Crystallogr. 44, 1272 (2011).

[35] T. Meier, F. Trybel, G. Criniti, D. Laniel, S. Khandarkhaeva, E. Koemets, T. Fedotenko, K. Glazyrin, M. Hanfland, M. Bykov et al., Phys. Rev. B 102, 165109 (2020).

[36] D. Simonova, E. Bykova, M. Bykov, T. Kawazoe, A. Simonov, N. Dubrovinskaia, and L. Dubrovinsky, Minerals 10, 1055 (2020).

[37] T. Meier, S. Khandarkhaeva, J. Jacobs, N. Dubrovinskaia, and L. Dubrovinsky, Appl. Phys. Lett. 115, 131903 (2019).

[38] T. Meier, D. Laniel, M. Pena-Alvarez, F. Trybel, S. Khandarkhaeva, A. Krupp, J. Jacobs, N. Dubrovinskaia, and L. Dubrovinsky, Nat. Commun. 11, 6334 (2020).

[39] Y. Akahama and H. Kawamura, J. Appl. Phys. 96, 3748 (2004).

[40] Y. Akahama and H. Kawamura, J. Appl. Phys. 100, 043516 (2006).

[41] C. Drechsel-Grau and D. Marx, Phys. Rev. Lett. 112, 148302 (2014).

[42] X. Xue and M. Kanzaki, J. Phys. Chem. B 111, 13156 (2007).

[43] S. Clough, A. Horsewill, P.J. McDonald, and F.O. Zelaya, Phys. Rev. Lett. 55, 1794 (1985).

[44] A. Suzuki, Mineral. Mag. 73, 479 (2009). 